\title{
Photoluminescence and nonlinear optical phenomena in plasmonic random media-A review of recent works
}

\author{
Cid B. de Araújo ${ }^{\text {a,* }}$, Luciana R.P. Kassab ${ }^{b}$, C. Tolentino Dominguez ${ }^{\mathrm{d}}$, Sidney J.L. Ribeiro ${ }^{\mathrm{c}}$, \\ Anderson S.L. Gomes ${ }^{a}$, Albert S. Reyna ${ }^{a}$
}

a Departamento de Física, Universidade Federal de Pernambuco, 50670-901 Recife, PE, Brazil

${ }^{\mathrm{b}}$ Faculdade de Tecnologia de São Paulo (FATEC-SP, CEETEPS), 01124-060 São Paulo, SP, Brazil

${ }^{\mathrm{c}}$ Institute of Chemistry, São Paulo State University (UNESP), 14801-970 Araraquara, SP, Brazil

d Laboratório de Óptica Biomédica e Imagem, Universidade Federal de Pernambuco, Recife 50740-530, PE, Brazil

\section{A R T I C L E I N F O}

Available online 3 December 2014

Keywords:

Photoluminescence

Rare-earth ions

Metallic nanoparticles

Nonlinear optics

\begin{abstract}
A B S T R A C T
Photoluminescence properties and nonlinear optical response of metal-dielectric nanocomposites (MDNCs) - germanate glasses, bio-cellulose membranes and colloids containing either silver (Ag) or gold $(\mathrm{Au})$ nanoparticles (NPs)-are reviewed. The phenomena discussed are: $i$. the photoluminescence enhancement observed from rare-earth doped $\mathrm{PbO}-\mathrm{GeO}_{2}$ glass containing Ag NPs; $i$. optical amplification at $1530 \mathrm{~nm}$ in RIB waveguides made with $\mathrm{PbO}-\mathrm{GeO}_{2}$ thin films covered with Au NPs; iii. Random Laser emission from a bio-cellulose membrane infiltrated with Rhodamine $6 \mathrm{G}$ and containing Ag NPs; $i v$. the nonlinearity management of high-order processes in colloids containing Ag NPs suspended in acetone. In all discussed cases the influence of the metallic NPs is clearly demonstrated and a procedure to control the nonlinear propagation of light beams in heterogeneous media is presented.
\end{abstract}

(c) 2014 Elsevier B.V. All rights reserved.

\section{Introduction}

The linear and nonlinear (NL) optical properties of metal-dielectric nanocomposites (MDNCs) have been largely investigated in the recent years. For instance, inorganic glasses, polymers and liquid colloids containing metallic nanoparticles (NPs) were studied under excitation by coherent and incoherent light sources. Therefore, a large number of new systems and new phenomena were identified [1-7]. One of the reasons for the interest in MDNCs is their large potential to be used in devices such as displays, light modulators, optical amplifiers and sensors. In general, it is required that photonic MDNCs present large transmittance and high optical response. However, when the incident light or the photoluminescence (PL) wavelengths are near the localized surface plasmon resonance of the NPs, an enhancement of the optical properties may be observed but the optical transmittance decreases. Then, the effective improvement when enhanced optical properties and thermal losses are considered is an important issue concerning the MDNC selection. Therefore, the choice of the appropriate light wavelength, the host material for the metallic NPs as well as their nature and concentration, are important aspects to consider aiming the optimization of a MDNC for a specific application.

The optical behavior of MDNCs can be controlled by adjusting the volume fraction, $f$, of the NPs with respect to the total volume

\footnotetext{
* Corresponding author.

E-mail address: cid@df.ufpe.br (C.B. de Araújo).
}

of the sample. For instance, large NL susceptibilities of colloids based on silver and gold NPs, excited by lasers operating in the 400-1000 nm range, were demonstrated for different values of $f[2,4-8]$. On the other hand many studies were focused on rareearth ions (REI) doped glasses containing metallic NPs [1,3,9-13]. Among the studied glasses germanates were identified as good host for REI and metallic NPs. For example, PL enhancement up to $\sim 1000 \%$ [12] and efficient frequency upconversion processes [13] were reported. Organic-inorganic hybrid MDNCs (polymers containing metallic NPs) were also exploited to improve the operation of Random Lasers [14,15].

In the present paper we review some of our recent works in this area. The optical behavior of a germanate glass, a bio-cellulose membrane and liquid colloids, containing either $\mathrm{Ag}$ or $\mathrm{Au} \mathrm{NPs}$, is discussed. Advances in the understanding and exploitation of PL and NL optical phenomena influenced by the presence of metallic NPs are discussed.

\section{Samples preparation}

The photonic MDNCs studied were prepared using chemical and physical techniques described in this section.

\subsection{Germanate glasses doped with rare-earth ions and metallic NPs}

$\mathrm{PbO}-\mathrm{GeO}_{2}$ glasses (labeled as PGO glasses), pure and doped with rare-earth ions (REI) and silver NPs, were prepared by the 
melting/quenching method using high-purity reagents (99.999\%) contained in alumina crucibles. After cooling the samples to room temperature inside the furnace, they were polished, cut, and then submitted to additional heat-treatment (HT) to nucleate silver NPs. Samples without metallic NPs were also fabricated to be used as reference (details on the PGO samples preparation are given in [11]). To investigate the size and shape of the NPs, a transmission electron microscope (TEM) operating at $300 \mathrm{kV}$ was used. A spectrophotometer was employed to measure the absorption spectra of the samples. PL spectra were measured with different excitation sources depending on the doping species. Thin PGO films with metallic NPs were also prepared using radio-frequency (RF) sputtering as reported [16]. For preparation of targets the oxide powders are mixed and then submitted to 8 ton uniaxial pressure, followed by sinterization at $750{ }^{\circ} \mathrm{C}$ during $10 \mathrm{~h}$. Targets composed by $\mathrm{Er}_{2} \mathrm{O}_{3}$ pellets (diameter: $1.0 \mathrm{~cm}$ ) positioned on $\mathrm{Yb}_{2} \mathrm{O}_{3}$ targets (diameter: $2.0 \mathrm{in}$.) were used to produce PGO films codoped with $\mathrm{Er}^{3+}$ and $\mathrm{Yb}^{3+}$. The films were deposited on a silica layer formed on a silicon substrate. The parameters used for fabrication are indicated in Table 1. RIB waveguides were obtained from the thin PGO films using optical lithography followed by the reactive ion etching process [17].

\subsection{Bio-cellulose $(B C)$ membranes doped with dyes and metallic NPs}

BC membranes were obtained from cultures of wild strains of Gluconacetobacter xylinus following a process described in $[18,19]$.

The samples for the experiments were obtained immersing the $\mathrm{BC}$ membranes for $24 \mathrm{~h}$ in $10 \mathrm{~mL}$ of a colloid containing Ag NPs. To infiltrate the gain medium, the $\mathrm{BC}$ membranes were immersed in $10 \mathrm{~mL}$ of an ethanol solution containing $10^{-4} \mathrm{M}$ of Rh6G and kept under strong stirring during $24 \mathrm{~h}$ at room temperature. Finally, the membranes were removed from the dye solution and placed in an oven at $40{ }^{\circ} \mathrm{C}$, during $2 \mathrm{~h}$, for evaporation of the ethanol.

To synthesize the Ag NPs, $20 \mathrm{~mL}$ of aqueous solution containing $\mathrm{AgNO}_{3}(0.25 \mathrm{mM})$ and sodium citrate $(0.25 \mathrm{mM})$ was prepared. Then, a solution of $0.6 \mathrm{~mL}$ of $\mathrm{NaBH}_{4}(0.1 \mathrm{M})$, that had been chilled in an icebath, was added. The final solution remained under strong stirring for $3 \mathrm{~h}$ to complete the reaction. After this procedure, the samples were submitted to a lyophilization for $24 \mathrm{~h}$.

\subsection{Liquid colloids with silver NPs}

Silver colloids were prepared by chemical synthesis as in [20] and submitted to photo-fragmentation using a Q-switched $\mathrm{Nd}$ : YAG laser ( $10 \mathrm{~Hz}, 8 \mathrm{~ns}, 85 \mathrm{~mJ} /$ pulse) for $1 \mathrm{~h}$.

This method has been applied by several authors and produces a homogeneous size distribution of NPs [21]. Spherical NPs with an average diameter $9.0 \pm 2.2 \mathrm{~nm}$ were obtained and samples with volume fraction $f$ from $0.5 \times 10^{-5}$ to $2.5 \times 10^{-5}$ were prepared.

Table 1

Parameters used for the PGO thin films fabrication.

Fabrication parameters

\begin{tabular}{ll}
\hline Base pressure (Torr) & $10^{-5}$ \\
Work pressure (mTorr) & $3.0-7.0$ \\
Oxigen flux (sccm) & 18 \\
RF power on the PGO target (W) & 60 \\
RF power on the $\mathrm{Er}_{2} \mathrm{O}_{3}-\mathrm{Yb}_{2} \mathrm{O}_{3}$ target (W) & 20 \\
Deposition time (h) & 12 \\
Distance target-substrate (cm) & 15 \\
\hline
\end{tabular}

\section{Results and discussion}

\subsection{Photoluminescence and optical amplification in glasses based MDNCS}

Efficient REI-based color displays were obtained by nucleation of Ag NPs in PGO glass triply-doped with $\mathrm{Tm}^{3+}, \mathrm{Er}^{3+}$ and $\mathrm{Yb}^{3+}$ ions. The Ag NPs were nucleated inside the samples by HT of $60 \mathrm{PbO}-40 \mathrm{GeO}_{2}$ (wt\%) glass containing $\mathrm{AgNO}_{3}$ (4.0 wt\%) $-\mathrm{Yb}_{2} \mathrm{O}_{3}$ (3.0 wt\%) $-\mathrm{Tm}_{2} \mathrm{O}_{3}(0.25 \mathrm{wt} \%)-\mathrm{Er}_{2} \mathrm{O}_{3}(0.5 \mathrm{wt} \%)$ in the starting composition. The formation of Ag NPs was verified using a TEM. When excited at $980 \mathrm{~nm}$, in resonance with the $\mathrm{Yb}^{3+}$ transition ${ }^{2} \mathrm{~F}_{7 / 2} \rightarrow{ }^{2} \mathrm{~F}_{5 / 2}$, the samples emit blue light at $477 \mathrm{~nm}$ (transition: ${ }^{1} \mathrm{G}_{4} \rightarrow{ }^{3} \mathrm{H}_{6}$ ) due to the $\mathrm{Tm}^{3+}$ ions, green light at $530 \mathrm{~nm}$ (transition: ${ }^{2} \mathrm{H}_{11 / 2} \rightarrow{ }^{4} \mathrm{I}_{15 / 2}$ ) and $550 \mathrm{~nm}$ (transition: ${ }^{4} \mathrm{~S}_{3 / 2} \rightarrow{ }^{4} \mathrm{I}_{15 / 2}$ ) due to the $\mathrm{Er}^{3+}$ ions, red light at $652 \mathrm{~nm}$ (transition: ${ }^{1} \mathrm{G}_{4} \rightarrow{ }^{3} \mathrm{~F}_{4}$ ) due to $\mathrm{Tm}^{3+}$ and at $660 \mathrm{~nm}$ (transition: ${ }^{4} \mathrm{~F}_{9 / 2} \rightarrow{ }^{4} \mathrm{I}_{15 / 2}$ ) due to the $\mathrm{Er}^{3+}$ ions. The relative intensity of the spectral bands depends on the NPs concentration. The silver NPs contribute for the PL intensity enhancement that was $60 \%$ larger than from a sample having the same composition but without silver NPs. This was the first time that the frequency upconversion enhancement due to metallic NPs is investigated in a triply REI doped glass. The results in the bluegreen region of the CIE chromaticity diagram are illustrated in Fig. 1(a). The behavior of the PL spectra as a function of the light intensity and the HT of the samples was published in [22].

The influence of Au NPs on the $1530 \mathrm{~nm}$ optical gain in $\mathrm{Er}^{3+} /$ $\mathrm{Yb}^{3+} \mathrm{PGO}-\mathrm{GeO}_{2} \mathrm{RIB}$-waveguides was also studied. The waveguides prepared are represented by Fig. 1(b) and (c). The RIB-waveguides were covered by layers of gold NPs obtained by RF sputtering with 5$7 \mathrm{~W}$ applied to a gold target using $5 \mathrm{mTorr}$ of argon during $15 \mathrm{~min}$. The propagation losses, measured at $1050 \mathrm{~nm}$, were smaller than $2 \mathrm{~dB} / \mathrm{cm}$ for waveguides varying from $3 \mu \mathrm{m}$ to $10 \mu \mathrm{m}$ width. The nanostructured gold films enhanced the $\mathrm{Er}^{3+}$ ions PL by $\sim 400 \%$ in the red region and $\sim 30 \%$ in the infrared, when the waveguide is excited at $980 \mathrm{~nm}$. The optical gain at $1530 \mathrm{~nm}$ was enhanced from $3 \mathrm{~dB}$ to $6.5 \mathrm{~dB}$ due to the local field growth in the vicinity of the NPs. In the waveguides covered with gold NPs the propagation mode is partially deviated to the interface between the dielectric film and the Au layer. Considering that the spatial extension of influence of the Au layer is $\approx 20 \mathrm{~nm}$, only $1.3 \%$ of the PGO thin film was influenced by the Au NPs. Consequently a large part of the optical field was confined to the PGO core and the losses due to ohmic dissipation in the Au layer were small [23]. The results demonstrate that the PGO RIB-waveguides, with or without $\mathrm{Au}$ NPs, are promising for integrated photonics.

\subsection{Random Laser based on BC membranes doped with a dye and metallic NPS}

Among photonic applications that exploit nanocomposites, Random Lasers (RLs) have been demonstrated and characterized in several combinations of scatterers and host materials. The optical feedback in RLs is provided by scatterer particles embedded in the active medium, or materials that can act as scatter and gain medium itself. As reported in [24], where further references to the subject of RLs can be found, we exploited samples of $\mathrm{BC}$ membranes doped with dyes and metallic NPs described in Section 2.2 to construct and characterize a flexible BC membrane based RL. The second harmonic of a pulsed Nd:YAG laser beam ( $532 \mathrm{~nm}, 6 \mathrm{~ns}$ ) was used as the excitation source, which was incident on the sample, with a $3 \mathrm{~mm}$ diameter beam spot size. Measurements were performed at low repetition rates (max $10 \mathrm{~Hz})$ and at single-shot illumination. PL signals and RL emission detected at $45^{\circ}$ with respect to the membrane surface was analyzed using a CCD coupled monochromator with $0.1 \mathrm{~nm}$ spectral resolution. Fig. 2 (a) shows the emitted intensity behavior versus input energy for the BC based RL using silver NPs to provide feedback. The inset shows a BC 
a

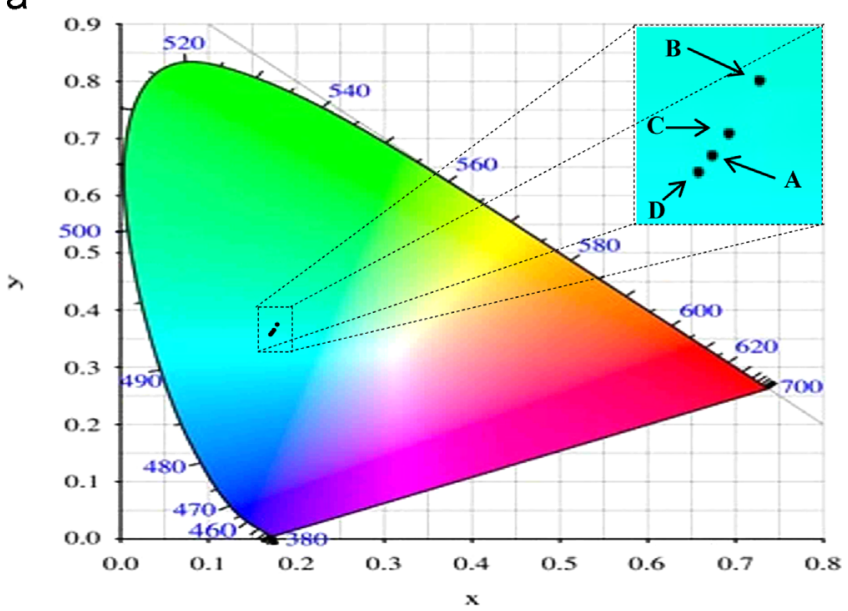

b

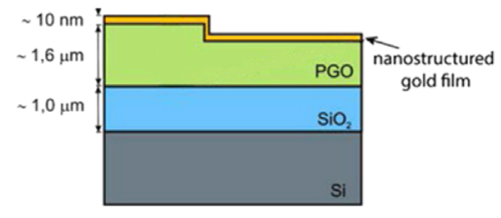

C

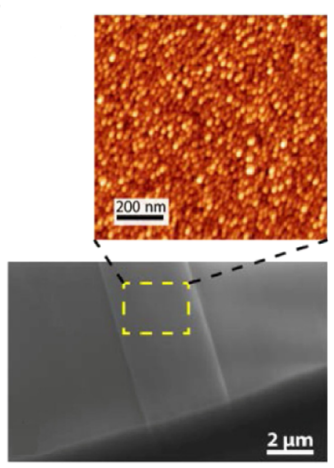

Fig. 1. (a) CIE chromaticity diagram. The four points in the figure correspond to: A-sample without NPs $(x=0.174 ; y=0.361)$; B-sample heat-treated during $12 \mathrm{~h}$ $(x=0.180 ; y=0.376) ; C-$ sample heat-treated during $24 \mathrm{~h}(x=0.176 ; y=0.366)$; D-sample heat-treated during $48 \mathrm{~h}(x=0.172 ; y=0.358)$. (b) Schematics showing the waveguide arrangement. (c) SEM micrograph and AFM image.

membrane doped with Rh6G. It can be observed from Fig. 2(a), that in the absence of NPs (green stars), the emission is characteristic of normal PL, whereas in the presence of the NPs (blue dots), a clear threshold exists around $1.8 \mathrm{~mJ}$, after which the intensity grows nonlinearly. The RL behavior is corroborated in Fig. 2(b), where the emission linewidth is plotted as a function of excitation energy. Without NPs (green stars) the bandwidth stays constant around $50 \mathrm{~nm}$, whilst with Ag NPs it is observed a bandwidth reduction to $\approx 6 \mathrm{~nm}$ for excitation above the threshold. The inset shows the image of the excited dye-doped BC sample without NPs (left picture) and with NPs (right picture) for excitation at $3.25 \mathrm{~mJ}$. Further details and discussion can be found in [24], where it is clear that the role of the Ag NPs deserve a deeper study, since for instance energy transfer between the dye molecules and the Ag NPs due to increased concentration can compete with stimulated emission and can reduce the RL efficiency.

\subsection{Nonlinearity management of metal-colloids}

The optical response of silver colloids was studied under different experimental conditions and NL susceptibilities associated to high-order processes were measured [6-8]. The results support the a

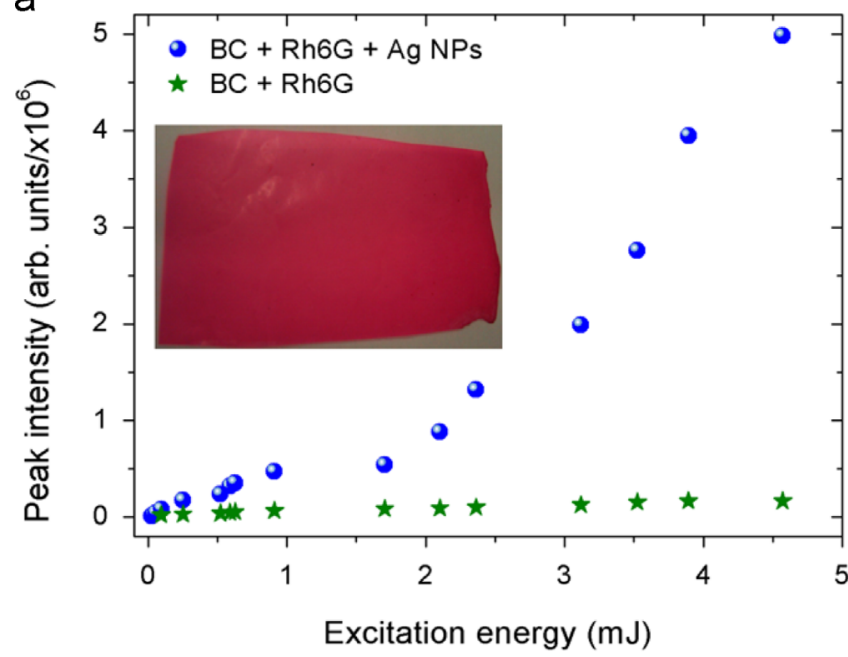

b

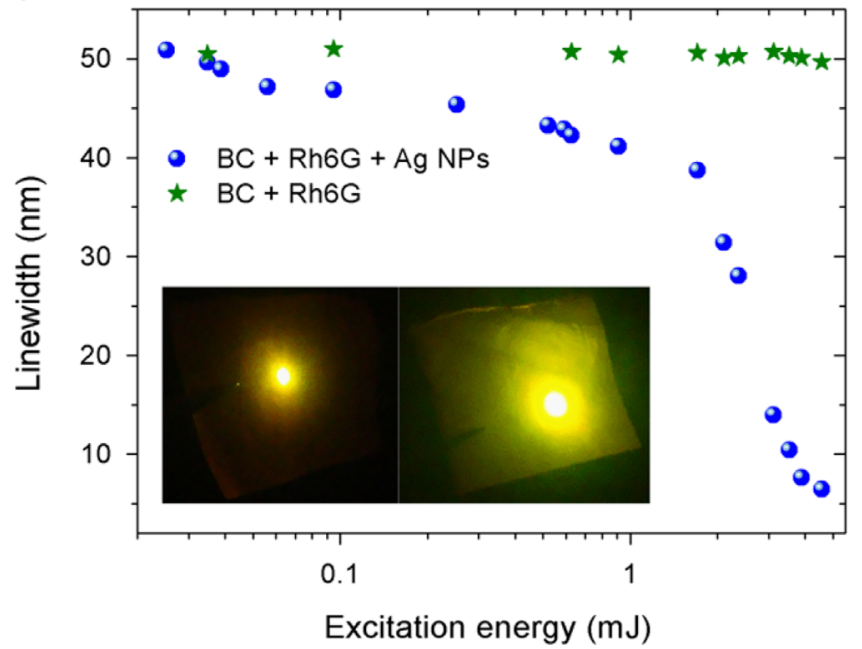

Fig. 2. (a) Peak intensity versus excitation pulse energy. Inset: $B C$ membrane doped with Rh6G $\left(10^{-4} \mathrm{M}\right)$. (b) Emission linewidth versus excitation pulse energy. Inset: Digital image of emission of a BC membrane doped with Rh6G without Ag NPs (left) and with Ag NPs (right). The excitation pulse energy was $3.52 \mathrm{~mJ}$.

description of the medium through effective NL susceptibilities of $(2 N+1)$ order, $\chi_{e f f}^{(2 N+1)}$, with $N=1,2,3 \ldots$, that include contributions from the NPs and the host liquid. For colloids with small NPs volume fraction, $f \ll 1$, and NPs dimensions smaller than the light wavelength, the MDNC can be described by the Maxwell-Garnet model extended to include high-order susceptibilities $[25,26] \cdot \chi_{\text {eff }}^{(2 N+1)}$ can be controlled by changing $f$ and the light intensity incident on the samples, following the nonlinearity management procedure described in $[25,26]$. For instance, new effects such as spatial-phase modulation due to $\chi_{\text {eff }}^{(5)}$ and $\chi_{\text {eff }}^{(7)}$, with suppressed refractive effect due to $\chi_{e f f}^{(3)}$, were demonstrated $[25,26]$.

Here we report preliminary results applying the nonlinearity management procedure presented in $[25,26]$ to control the formation and propagation of spatial solitons in colloids. Varying the volume fraction of Ag NPs suspended in acetone it was possible to manage the NL response to obtain $\operatorname{Re} \chi_{\text {eff }}^{(3)}=0, \operatorname{Re} \chi_{\text {eff }}^{(5)}>0$ and $\operatorname{Re} \chi_{\text {eff }}^{(7)}<0$, and it was observed the propagation of $(2+1)$-dimensional, $(2+1)$-D, bright spatial solitons.

Fig. 3(a) shows a scheme of the setup used to monitor the propagation of the laser beam through the samples. A Nd: YAG laser ( $80 \mathrm{ps}, 532 \mathrm{~nm}$, single pulses at $10 \mathrm{~Hz}$, maximum pulse energy: $10 \mu \mathrm{J}$ ) 
a

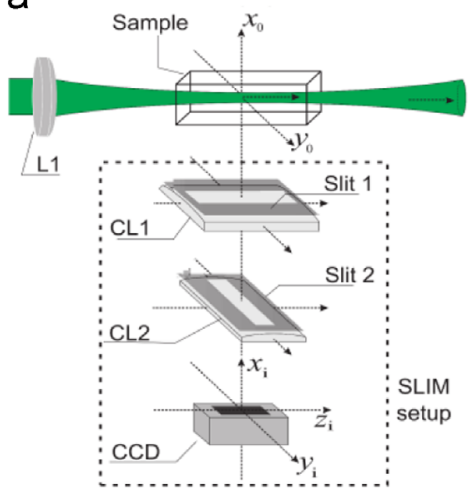

C

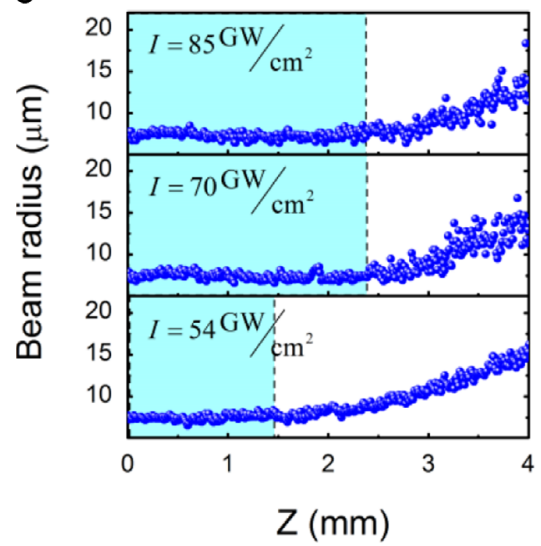

b

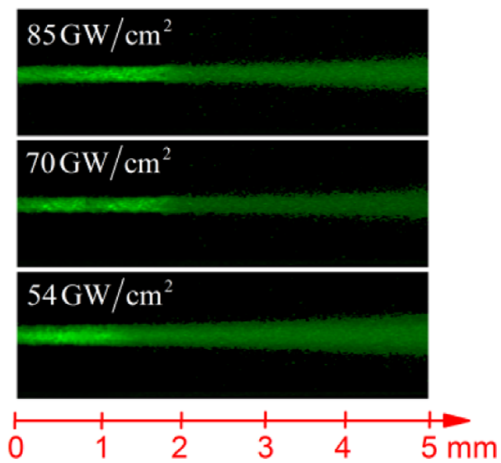

d

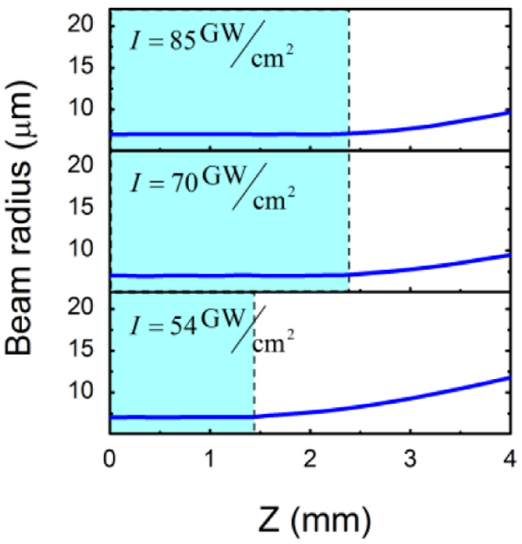

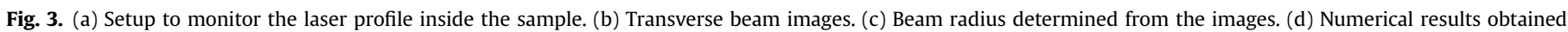
solving the propagation equation corresponding to $f=1.6 \times 10^{-5}$. The shaded areas indicate the regions of stable soliton propagation.

was employed and a CCD camera was used to record the light scattered by the NPs along a direction perpendicular to the beam axis. The data were analyzed using the Scattered Light Imaging Method-SLIM [27]. From the images recorded we could determine the beam radius for different positions inside the sample and compare with the results predicted by the propagation equation.

Fig. 3(b) shows the variation of the laser beam radius along the pathway inside the sample when $f=1.6 \times 10^{-5}$, determined from the images of Fig. 3(a), using SLIM. Notice that as the laser intensity increases, the propagation distance at which the beam waist remains constant also increases. Maximum propagation distance of $2.3 \mathrm{~mm}$ corresponding to $\sim 10$ Rayleigh lengths was observed at the highest input intensities. The NL propagation of the light beam was described by: $2 i k(\partial E / \partial z)+\Delta E=-\left(\omega^{2} / c^{2}\right)\left[3 \chi_{\text {eff }}^{(3)}|E|^{2} E+10 \chi_{\text {eff }}^{(5)}|E|^{4} E+\right.$ $\left.35 \chi_{\text {eff }}^{(7)}|E|^{6} E\right]$, where $E$ is the optical field amplitude, $\Delta$ is the transverse Laplacian operator, $z$ is the propagation direction, $k=2 \pi n_{0} / \lambda, \lambda$ is the laser wavelength, $n_{0}$ is the linear refractive index, $\omega$ is the laser frequency and $c$ is the speed of light in vacuum. The expression inside brackets represents the total effective NL susceptibility and the numerical coefficients are due to the degeneracy factors for the $i$ th order process [28]. The effective susceptibilities $\chi_{\text {eff }}^{(2 N+1)}, N=1-3$, depend on $f$ and their values were determined using the Z-scan technique $[6,29]$. The good agreement between the experimental and theoretical results can be observed comparing the shaded rectangles drawn in Fig. 3(c) and (d).

\section{Summary}

The MDNCs presented, based on glasses doped with REI and bio-cellulose membranes infiltrated with a laser dye, exhibit large potential for photonic devices such as displays, optical amplifiers and Random Lasers. A procedure to manage the NL response of MDNCs was also presented that indicates a way for fabrication of materials with high-order NL response appropriate for particular applications.

\section{Acknowledgments}

We acknowledge financial support by the Brazilian agencies Conselho Nacional de Desenvolvimento Científico e Tecnológico (CNPq), Fundação de Amparo a Ciência e Tecnologia do Estado de Pernambuco (FACEPE), and Fundação de Amparo a Pesquisa do Estado de São Paulo (FAPESP). The work was performed in the framework of the National Institute of Photonics (INCT de Fotônica) and PRONEX/CNPq/FACEPE. The Laboratório de Microscopia Eletrônica (IFUSP) and the Centro de Tecnologias Estratégicas do Nordeste (CETENE) are acknowledged for the TEM images.

\section{References}

[1] M. Yamane, Y. Asahara, Glasses for Photonics, Cambridge University Press, Cambridge, UK, 2000.

[2] C. Flytzanis, J. Phys. B: At. Mol. Opt. Phys. 38 (2005) 8661.

[3] J.P. Huang, K.W. Yu, Phys. Rep. 431 (2006) 87.

[4] K.-H. Kim, A. Husakou, J. Herrmann, Opt. Express 18 (2010) 7488.

[5] Plasmonics, Nature Photonics, Focus issue: 6 (2012) 707.

[6] E.L. Falcão-Filho, C.B. de Araújo, J.J. Rodrigues Jr. J. Opt. Soc. Am. B 24 (2007) 2948.

[7] E.L. Falcão-Filho, R. Barbosa-Silva, R.G. Sobral-Filho, A.M. Brito-Silva, A. Galembeck, C.B. de Araújo, Opt. Express 18 (2010) 21636.

[8] J. Jayabalan, A. Singh, S. Khan, R. Chari, J. Appl. Phys. 112 (2012) 103524.

[9] O.L. Malta, P.A.S. Cruz, G.F. de Sá, F. Auzel, J. Lumin. 33 (1985) 261.

[10] O.L. Malta, Phys. Lett. 114 (1986) 198.

[11] C.B. de Araújo, D.S. da Silva, T.A.A. de Assumpção, L.R.P. Kassab, D.M. da Silva, Sci. World J. 2013 (2013) (Article no. 385193).

[12] L.R.P. Kassab, D.S. da Silva, C.B. de Araújo, J. Appl. Phys. 107 (2010) 113506. 
[13] D.S. da Silva, T.A.A. de Assumpção, L.R.P. Kassab, C.B. de Araújo, J. Alloys Compd. 586 (2014) S516.

[14] C.T. Dominguez, R.L. Maltez, R.M.S. dos Reis, L.S.A. de Melo, C.B. de Araújo, A.S. L. Gomes, J. Opt. Soc. Am. B 28 (2011) 1118.

[15] X. Meng, K. Fujita, S. Murai, K. Tanaka, Phys. Rev. A 79 (2009) 053817.

[16] D.M. da Silva, L.R.P. Kassab, J.R. Martinelli, C.B. de Araújo, J. Non-Cryst. Solids 356 (2010) 2602

[17] V.D. Del Cacho, D.M. da Silva, L.R.P. Kassab, A.L. Siarkowski, N.J. Morimoto, . Alloys Compd. 509 (2011) S434

[18] G.F. Perotti, H.S. Barud, S.J.L. Ribeiro, V.R.L. Constantino, J. Braz. Chem. Soc. 25 (2014) 1647

[19] S. Saska, L.N. Teixeira, P.T. Oliveira, A.M.M. Gaspar, S.J.L. Ribeiro, Y. Messaddeq, R. Marchetto, J. Mater. Chem. 22 (2012) 22102.

[20] P.C. Lee, D. Meisel, J. Phys. Chem. 86 (1982) 3391

[21] A.M. Brito-Silva, L.A. Gómez, C.B. de Araújo, A. Galembeck, J. Nanomater. 2010 (2010) 142897.
[22] M.E. Camilo, T.A.A. de Assumpção, D.M. da Silva, D.S. da Silva, L.P.R. Kassab C.B. de Araújo, J. Appl. Phys. 113 (2013) 153507.

[23] D.M. da Silva, L.P.R. Kassab, A.L. Siarkowoski, C.B. de Araújo, Opt. Express 22 (2014) 16424.

[24] M.V. dos Santos, C.T. Dominguez, J.V. Schiavon, H.S. Barud, L.S.A. de Melo, S.J.L. Ribeiro, A.S.L. Gomes, C.B. de Araújo, J. Appl. Phys. 115 (2014) 083108.

[25] A.S. Reyna, C.B. de Araújo, Phys. Rev. A 89 (2014) 063803.

[26] A.S. Reyna, C.B. de Araújo, Opt. Express 22 (2014) 22456.

[27] K.C. Jorge, R. Riva, J.M.S. Sakamoto, N.A.S. Rodrigues, M.G. Destro, Appl. Opt. 53 (2014) 4555

[28] P.N. Butcher, D. Cotter, The Elements of Nonlinear Optics, Cambridge University Press, Cambridge, UK, 1990.

[29] M. Sheik-Bahae, A.A. Said, T. Hei, D.J. Hagan, E.W. Van Stryland, IEEE J. Quantum Electron. 26 (1990) 760. 